\title{
Age-Related Pulmonary Crackles (Rales) in Asymptomatic Cardiovascular Patients
}

\author{
Hajime Kataoka, $M D^{1}$ \\ Osamu Matsuno, $\mathrm{MD}^{2}$ \\ 'Division of Internal Medicine, \\ Nishida Hospital, Oita, Japan \\ ${ }^{2}$ Division of Respiratory Disease, Oita \\ University Hospital, Oita, Japan
}

\begin{abstract}
PURPOSE The presence of age-related pulmonary crackles (rales) might interfere with a physician's clinical management of patients with suspected heart failure. We examined the characteristics of pulmonary crackles among patients with stage A cardiovascular disease (American College of Cardiology/American Heart Association heart failure staging criteria), stratified by decade, because little is known about these issues in such patients at high risk for congestive heart failure who have no structural heart disease or acute heart failure symptoms.
\end{abstract}

METHODS After exclusion of comorbid pulmonary and other critical diseases, 274 participants, in whom the heart was structurally (based on Doppler echocardiography) and functionally (B-type natriuretic peptide $<80 \mathrm{pg} / \mathrm{mL}$ ) normal and the lung (X-ray evaluation) was normal, were eligible for the analysis.

RESULTS There was a significant difference in the prevalence of crackles among patients in the low (45-64 years; $n=97 ; 11 \% ; 95 \% \mathrm{Cl}, 5 \%-18 \%)$, medium (65-79 years; $n=121 ; 34 \% ; 95 \% C l, 27 \%-40 \%)$, and high (80-95 years; $n=56 ; 70 \%$; $95 \% \mathrm{Cl}, 58 \%-82 \%)$ age-groups $(P<.001)$. The risk for audible crackles increased approximately threefold every 10 years after 45 years of age. During a mean follow-up of $11 \pm 2.3$ months $(n=255)$, the short-term ( $\leq 3$ months) reproducibility of crackles was $87 \%$. The occurrence of cardiopulmonary disease during follow-up included cardiovascular disease in 5 patients and pulmonary disease in 6.

CONCLUSIONS Recognition of age-related pulmonary crackles (rales) is important because such clinically unimportant crackles are so common among elderly patients that, without knowledge of this phenomenon, their existence might interfere with the physician's management of cardiopulmonary patients.

Ann Fam Med 2008;6:239-245. DOI: 10.1370/afm.834.

\section{INTRODUCTION}

$\mathrm{H}$ eart failure is a common problem, especially in elderly patients. ${ }^{1}$ The appearance of pulmonary crackles (rales), defined as discontinuous, interrupted, explosive respiratory sounds during inspiration, is one of the most important signs of heart failure deterioration. ${ }^{2}$ Many older patients with asymptomatic cardiovascular disease seem to have pulmonary crackles, even in the absence of apparent cardiac dysfunction or comorbid pulmonary disease. The prevalence and pathologic importance of crackles in apparently normal persons are controversial..$^{3-5}$ Moreover, little is known about these issues in patients with asymptomatic cardiovascular disease. Frequent age-related pulmonary crackles might interfere with the physician's management of patients with suspected heart failure or presumed pulmonary disease. Because diagnostic and therapeutic decisions may be made on the basis of the finding of crackles, knowledge of their occurrence would be clinically important. In this study we examined the characteristics of pulmonary crackles among adult patients, stratified by decade, with stage A cardiovascular disease status as defined by the American College of Cardiology/American Heart Association 2001 chronic heart failure guidelines, ${ }^{6}$ ie, patients at high risk for congestive 
heart failure, but without structural heart disease or symptoms of heart failure, who were also free from comorbid pulmonary disease.

\section{METHODS}

\section{Study Design and Patients}

This prospective study was performed in the cardiology outpatient clinic of Nishida Hospital (Oita, Japan) between June 2005 and November 2006. Adult outpatients (aged 45 years and older) undergoing treatment for cardiovascular disease, including metabolic syndrome, were first screened by research nurses, who obtained consent and recorded a brief cardiac and pulmonary history and current complaints. Patients were not eligible for this investigation if they reported acute cardiopulmonary complaints or if they had a current diagnosis or history of structural heart disease, decompensated heart failure, chronic pulmonary disease, or recent episode (less than 3 months) of acute respiratory disease. No patient was suspected either clinically or after examination or investigation to suffer from connective tissue diseases

All screened patients had a physical examination, blood chemistry tests, a 12-lead electrocardiogram (ECG), and a simple chest radiograph. At this second step, patients were excluded if they had high serum creatinine levels $(\geq 1.2 \mathrm{mg} / \mathrm{dL})$, abnormal ECG rhythm (atrial fibrillation or left bundle branch block), or radiographic abnormalities. Finally, all the patients screened at the second step underwent a cardiac Doppler echocardiogram and blood sampling for analysis of serum B-type natriuretic peptide (BNP) levels. At this final step, patients were excluded if they had abnormal findings on a Doppler echocardiogram ${ }^{7,8}$ or high BNP levels ( $\geq 80 \mathrm{pg} / \mathrm{mL}$ ). ${ }^{9,10}$

Because the sensitivity of a simple chest radiograph alone might be insufficient for assessing the structural normalcy of lung tissue, some study patients underwent thoracic high-resolution computed tomography $(\mathrm{CT}) .{ }^{11-13}$

We observed a substantial number of participants from 6 to 12 months. During this follow-up period, the short-term (3 or fewer months) reproducibility of the presence or absence of pulmonary crackles and the occurrence of critical cardiovascular or pulmonary disease were evaluated. The protocol was approved by the ethics committee of Nishida Hospital, and all patients provided informed consent.

\section{Evaluation by Auscultation}

A single senior cardiologist (H.K.) auscultated carefully the anterior to posterior basilar sites in each hemithorax in seated patients who were asked to per- form periodic slow deep respiration. The cardiologist who performed the auscultation was blinded to chest radiograph, Doppler echocardiogram, and high-resolution CT findings. Pulmonary crackles were defined as discontinuous, interrupted explosive sounds during inspiration. ${ }^{14}$ Audible crackles were classified as fine or coarse crackles according to their dominant acoustic nature. ${ }^{14}$ Some previous studies reported that basilar crackles are often heard during the first few deep breaths, even in apparently normal persons. ${ }^{4,15}$ In our study, only inspiratory crackles that appeared recurrently during consecutive respiratory cycles were accepted as the presence of crackles. We graded such basal crackles from 0 to 4 based on the longitudinal extension from the lung base upward to the $\operatorname{apex}^{16}$ : a score of 1 indicates crackles over one-quarter of the lung height and a score of 4 indicate crackles over the entire lung height. Additionally, we defined bilateral transverse extension of crackles over two-thirds of the hemithorax as diffuse crackles.

\section{Other Main Measurements}

We obtained high-resolution thoracic CT scans ${ }^{12,13}$ with 1-mm collimation, $130 \mathrm{kVp}$ and $200 \mathrm{~mA}$, by using a high-frequency reconstruction algorithm (X-Vision/ GX, Toshiba Co, Ltd, Tokyo, Japan). A single senior pneumologist (O.M.) who was blinded to other clinical tests evaluated the CT image. Serum BNP was measured using the Shionoria assay method. ${ }^{17}$ The Doppler echocardiogram was performed using a commercially available real-time, wide-angle phased-array system (Aloka SSD-2000, Aloka Co, Ltd, Tokyo, Japan).

\section{Statistical Analysis}

Continuous variables are expressed as mean values plus or minus standard deviations, and categorical data are expressed as frequencies and percentages. We assessed differences among the 3 study groups stratified arbitrarily by age by using 1-way analysis of variance for continuous variables with Bonferroni's correction for multiple comparisons; they were analyzed using the Kruskal Wallis $\mathrm{H}$ test for categorical data and the Mann-Whitney $U$ test with Bonferroni's correction for multiple comparisons as the post hoc test. We used a 2 -tailed unpaired Student's $t$ test and a $\chi^{2}$ test for comparisons between patients with and without pulmonary crackles. Using logistic regression analysis, we determined the presence or absence of pulmonary crackles by taking univariate predictors of the appearance of crackles and using iterative modeling procedures to arrive at the most efficient model. The threshold for entry of variables into the model was $P<.10$. We calculated odds ratios (OR) and 95\% confidence intervals $(\mathrm{CI})$, and a 2 -tailed $P$ value of $<.05$ was considered sig- 
nificant. Statistical analyses were performed using SAS Statistical Software, version 8.2 (SAS Institute, Inc, Cary, North Carolina).

\section{RESULTS}

\section{Patients Selection}

Of the 385 study participants with current treatment for hypertension, diabetes, or dyslipidemia, 52 were excluded because of high serum creatinine levels $(n=7)$, abnormal ECG rhythm $(n=13)$, chest radiograph abnormality of the lower lung $(n=15)$, or any combination of these $(n=17)$. Chest radiograph abnormalities consisted of localized infiltration or reticular opacities in the lower lung field in 16, pleural thickening in 3 , and both in 4 . After exclusion, the remaining 333 patients underwent a cardiac Doppler echocardiography and measurement of serum BNP levels. At this step, a total of 59 patients were excluded from the analysis because of abnormal Doppler echocardiogram findings $(n=11)$, high BNP levels $(n=14)$, or both $(n=34)$. Doppler echocardiogram abnormalities included left ventricular systolic dysfunction (ejection fraction $<50 \%$ ) in 9 patients, segmental wall motion abnormality in 4 , hypertrophy of the septal or left ventricular posterior wall $(\geq 12 \mathrm{~mm})$ in 10 , left ventricular dilatation $(\geq 140 \mathrm{~mL})$ in 14 , abnormal peak early-to-atrial velocity (EA) ratio of Doppler-derived left ventricular diastolic dysfunction in 13 , and major valvular regurgitation in 27 ( 2 or more abnormali- ties in 18 patients). After this final exclusion process, 274 asymptomatic adult patients with sinus rhythm in whom the heart was assumed to be structurally normal (determined by Doppler echocardiography) and functionally normal (determined by serum BNP), and with structurally normal lungs as evaluated by a simple radiograph, remained eligible for analysis.

\section{Clinical Characteristics}

Table 1 displays the baseline clinical characteristics of the study participants and the 3 categories stratified by age-groups (low $=45-64$ years; $n=97$, medium $=65$ 79 years; $\mathrm{n}=121$; and high $=80-95$ years; $\mathrm{n}=56$ ). Men made up $28 \%$ of the participants. There were significant differences in demographic features across the 3 age-groups. The higher the age-group, the more likely patients were to have venous insufficiency or edema in the lower leg and higher mean values of serum BNP and creatinine. The prevalence of a smoking habit was not different across the 3 age-groups.

\section{Characteristics of Pulmonary Crackles by Age}

The overall prevalence of audible pulmonary crackles was 92 of 274 study patients $(34 \% ; 95 \%$ CI, 31\%-36\%). Among these 92 patients with audible crackles, 79 patients $(86 \%)$ had predominantly fine crackles, and the remaining 13 patients (14\%) had predominantly coarse crackles. Table 2 displays the prevalence of audible pulmonary crackles across the 3 age-groups The higher the age-group, the more frequently the

Table 1. Demographic Characteristics of Study Patients Classified by Age-Group

\begin{tabular}{|c|c|c|c|c|}
\hline Characteristics & $\begin{array}{l}\text { All Patients } \\
(n=274)\end{array}$ & $\begin{array}{l}\text { Low (45-64 Years) } \\
(n=97)\end{array}$ & $\begin{array}{l}\text { Medium (65-79 Years) } \\
\text { ( } \mathrm{n}=121)\end{array}$ & $\begin{array}{l}\text { High (80-95 Years) } \\
(n=56)\end{array}$ \\
\hline Age, years & $69.2 \pm 12$ & $56 \pm 9.8$ & $73.1 \pm 4.1$ & $83.7 \pm 3.5$ \\
\hline Men, No. (\%) & $77(28)$ & $34(35)$ & $28(23)$ & $15(27)$ \\
\hline Current or previous smoker, No. (\%) & $48(18)$ & $21(22)$ & $18(15)$ & $9(16)$ \\
\hline \multicolumn{5}{|l|}{ Treatment } \\
\hline Hypertension, No. (\%) & $225(82)$ & $78(80)$ & $97(80)$ & $50(89)$ \\
\hline Dyslipidemia, No. (\%) & $87(32)$ & $34(35)$ & $45(37)$ & $8(14)$ \\
\hline Arrhythmia, No. (\%) & $39(14)$ & $16(16)$ & $14(12)$ & $9(16)$ \\
\hline Stable angina pectoris, No. (\%) & $26(9)$ & $4(4)$ & $16(13)$ & $6(11)$ \\
\hline Diabetes mellitus, No. (\%) & $24(8)$ & $9(9)$ & $9(7)$ & $6(11)$ \\
\hline Third heart sound, No. (\%) & $4(1)$ & $0(0)$ & $3(2)$ & $1(2)$ \\
\hline Venous insufficiency, No. $(\%)^{b}$ & $58(21)$ & $9(9)$ & $26(21)$ & $23(41)$ \\
\hline Leg edema, No. $(\%)^{c}$ & $33(12)$ & $6(6)$ & $15(12)$ & $12(21)$ \\
\hline Left ventricular ejection fraction, \% & $61.2 \pm 7.1$ & $61.9 \pm 6.5$ & $60.9 \pm 7.1$ & $60.8 \pm 7.8$ \\
\hline Serum creatinine, $\mathrm{mg} / \mathrm{dL}^{c}$ & $0.8 \pm 0.24$ & $0.73 \pm 0.21$ & $0.78 \pm 0.21$ & $0.8 \pm 0.23$ \\
\hline BNP, $\mathrm{pg} / \mathrm{mL}^{\mathrm{b}}$ & $31.6 \pm 19.1$ & $23.4 \pm 17.2$ & $33.1 \pm 18.3$ & $42.4 \pm 19.8$ \\
\hline $\begin{array}{l}\text { BNP }=\text { serum B-type natriuretic peptide. } \\
\text { a } P<.01 \text { among groups in low, medium, an } \\
\text { b } P<.001 \text { among groups in low, medium, a } \\
\text { ' } P<.05 \text { among groups in low, medium, an }\end{array}$ & $\begin{array}{l}\text { gh age-groups. } \\
\text { igh age-groups. } \\
\text { gh age-groups. }\end{array}$ & & & \\
\hline
\end{tabular}


patients had audible pulmonary crackles. Audible pulmonary crackles in such patients were often fine and localized to the lower quadrant of the lung field irrespective of the location, ie, anterior, lateral, or posterior on the thoracic wall. Increasing age was associated with the extension of crackles from the unilateral to bilateral hemithorax. Fourteen patients $(5 \%)$ had grade 2 crackles. Only 3 patients (1\%) had diffuse transverse and grade 2 longitudinal extensions of the pulmonary crackles. Other abnormal lung sounds heard at the basal lung included local wheeze in 3 patients

\section{CT Findings}

Thoracic high-resolution CT was performed in 55 participants who had audible crackles, including 35 of $39(90 \%)$ patients with bilateral crackles and 20 of 53 (38\%) patients with unilateral crackles. CT findings of the lower lung field appeared normal in 20 of 55 participants (36\%). Minimal or focal subpleural interstitial changes were detected in 27 patients (49\%), solitary septal thickening in $6(11 \%)$, and small nodules in 2 $(4 \%)$. One patient was suspected to have a pathologic pulmonary condition based on the CT findings, which indicated extensive bibasal fibrotic/reticular abnormalities. ${ }^{12,13}$ This patient was an 83 -year-old man who had diffuse and grade 2 pulmonary crackles and whose condition was diagnosed as unclassifiable interstitial pneumonia ${ }^{12,13}$ during the follow-up period.

Among 19 patients without crackles, focal subpleural interstitial changes on a CT scan were found in 3 patients (16\%), focal septal thickening in 1 (5\%), and small nodules in 1 (5\%). Of the 74 study patients examined by CT, none showed heart failure-related CT findings, ${ }^{11}$ such as pulmonary edema, pleural effusion, and venous engorgement.

\section{Predictors of Pulmonary Crackles}

Table 3 displays the comparison of the clinical variables between study patients with $(\mathrm{n}=92)$ and without $(\mathrm{n}=182)$ pulmonary crackles. A logistic regression analysis was performed for presence or absence of pulmonary crackles, with age, leg venous insufficiency, leg edema, serum creatinine, and serum BNP as independent variables. Age was the only independent predic-
Table 3. Comparison of Clinical Variables of Patients With and Without Pulmonary Crackles

\begin{tabular}{|c|c|c|c|}
\hline \multirow[b]{2}{*}{ Variable } & \multicolumn{2}{|c|}{ Pulmonary Crackles } & \multirow[b]{2}{*}{$\begin{array}{c}P \\
\text { Value }\end{array}$} \\
\hline & $\begin{array}{l}\text { Present } \\
(\mathrm{n}=92)\end{array}$ & $\begin{array}{c}\text { Absent } \\
(n=182)\end{array}$ & \\
\hline Age, years & $76.9 \pm 7.8$ & $65.3 \pm 11.2$ & $<.001$ \\
\hline Men, No. (\%) & $24(26)$ & $53(29)$ & NS \\
\hline $\begin{array}{l}\text { Current/previous } \\
\text { smoker, No. (\%) }\end{array}$ & $15(16)$ & $33(18)$ & NS \\
\hline $\begin{array}{l}\text { Third heart sound, } \\
\text { No. (\%) }\end{array}$ & $3(3)$ & $1(0.5)$ & NS \\
\hline $\begin{array}{l}\text { Venous insufficiency, } \\
\text { No. (\%) }\end{array}$ & $27(29)$ & $31(17)$ & $<.05$ \\
\hline Leg edema, No. (\%) & $17(18)$ & $16(9)$ & $<.05$ \\
\hline $\begin{array}{l}\text { Left ventricular ejection } \\
\text { fraction, \% }\end{array}$ & $61.1 \pm 7.1$ & $61.5 \pm 7.9$ & NS \\
\hline $\begin{array}{l}\text { Serum creatinine, } \\
\mathrm{mg} / \mathrm{dL}\end{array}$ & $0.79 \pm 0.21$ & $0.75 \pm 0.19$ & $<.1$ \\
\hline BNP, pg/mL & $36.4 \pm 19.6$ & $29.1 \pm 19.0$ & $<.01$ \\
\hline
\end{tabular}

Table 4. Predictors of Pulmonary Crackles Determined by Logistic Regression Analysis

\begin{tabular}{llll}
\hline Parameter & OR & 95\% Cl & P Value \\
\hline Age per 10 years & 3.23 & $2.22-4.70$ & $<.001$ \\
Venous insufficiency & 1.02 & $0.499-2.08$ & .961 \\
Leg edema & 1.43 & $0.597-3.41$ & .425 \\
Serum creatinine & 1.29 & $0.314-5.26$ & .727 \\
BNP & 0.997 & $0.981-1.01$ & .731 \\
BNP = serum B-type natriuretic peptide; Cl = confidence interval, \\
OR = odds ratio.
\end{tabular}

tor of the presence or absence of pulmonary crackles (Table 4). For the appearance of crackles, the adjusted OR of age per 10 years was 3.23 (95\% CI, 2.22-4.70; $P<.001)$. Other variables were not significant predic- 
tors of the presence or absence of pulmonary crackles. Leg edema was strongly associated with the presence of peripheral venous insufficiency in the study population $\left(\chi^{2}=27.2, P<.001\right)$.

\section{Follow-Up}

A clinical follow-up was obtained for 255 patients over a mean of $11 \pm 2.3$ months. At the short-term follow-up of $1.75 \pm 0.8$ months, the reproducibility of pulmonary crackles was $87 \%$. The occurrence of critical cardiopulmonary disease during the entire follow-up included congestive heart failure in 3 patients, acute coronary syndrome in 2, bacterial pneumonia in 5, and unclassifiable interstitial pulmonary pneumonia in 1. Among the 5 patients with cardiovascular events, 2 patients with crackles at study entry (diffuse and grade 2 crackles in 1 patient) developed congestive heart failure during follow-up, and the remaining 3 patients were free from crackles at study entry. Among 5 patients with subsequent complicating pneumonia, 2 patients had unilateral grade 1 pulmonary crackles, but the other 3 patients were free from crackles at study entry.

\section{DISCUSSION}

Our study has shown that elderly patients with stage A cardiovascular status ${ }^{6}$ frequently have audible pulmonary crackles, even in the absence of apparent cardiac dysfunction and comorbid pulmonary disease. Characteristically, crackles in such patients are fine and are almost always restricted to an area localized to the lower quadrant of the lung field. Diffuse basal crackles involving the bilateral hemithorax were exceptional phenomena in this study. These age-related crackles, except for the diffuse type, are not considered clinically significant over the medium-term follow-up; however, the possibility of new-onset heart failure or occult interstitial or other types of active lung disease must be kept in mind on subsequent evaluations.

The prevalence and pathologic significance of crackles in apparently normal persons is subject to controversy. ${ }^{3-5}$ Although some investigators report that crackles do not occur in normal persons, others disagree. The observations in this study are somewhat consistent with the findings of Cabot et $\mathrm{al}_{1}{ }^{3}$ who reported frequent detection of "crepitant rales" in middle-aged or elderly patients with "normal chests" examined during the first few breaths after shallow tidal breathing. Our findings show that the risk ratio of pulmonary crackles increases approximately threefold every 10 years after 45 years of age in patients with cardiovascular disease and apparently normal heart function. Minimal interstitial changes in some patients with crackles were found with high-resolution CT examination. ${ }^{12,13}$ Many of our older patients had likely been exposed to various environmental agents over the years, including probable infectious lung disease, which might contribute to the interstitial changes seen on CT or subsequent production of crackles. Such CT findings in the lung with age could be due to environmental pollutants rather than simply aging alone.

In patients with cardiovascular disease, physical examination is the primary step in evaluating the presence and severity of fluid retention in those with possible heart failure. ${ }^{2,6}$ The appearance of pulmonary crackles is among the most important signs of congestive heart failure. The accuracy of detecting crackles for diagnosing the deterioration of heart failure, however, varies greatly across studies: sensitivity $13 \%$ to $70 \%$, specificity $35 \%$ to $100 \%$, positive predictive value $19 \%$ to $100 \%$, negative predictive value $17 \%$ to $85 \% .{ }^{18}$ This variable accuracy among studies might be explained by at least 2 factors, ie, the population studied and the definition of crackles. ${ }^{19}$

First, the utility of a test is always determined by the nature of the population studied and can only be generalized if it is representative of other populations likely to be of interest. Thus, although pulmonary crackles ought to be a very specific sign, sometimes they are not, perhaps because crackles related to heart failure are so easily confused with those of interstitial fibrosis, pneumonia, and bronchitis. ${ }^{4}{ }^{14}$ Recognition of age-related crackles is important because such crackles are so common among apparently normal elderly patients with cardiovascular disease that their existence in the population of interest would additionally aggravate the specificity for diagnosing heart failure status.

Second, the criteria required for determining the crackles related to heart failure also affect the accuracy of the test results for heart failure diagnosis. Many previous studies, except several, ${ }^{16,20,21}$ lacked definite criteria for diagnosing the presence of crackles related to heart failure, and the mere presence or absence of the crackles was often expressed as the definition. The nature of the age-related crackles in our study, ie, restriction to the lower quadrant of the lung field, would partly support the notion of Stevenson et al ${ }^{16}$ that physical evidence of pulmonary congestion might be specific for grade 2 or 4 crackles, not for grade 1 , but such strict criteria resulted in low sensitivity for diagnosing congestive heart failure.

The physical signs of heart failure in the elderly are often difficult to interpret. ${ }^{22-24}$ Superimposition of changes caused by aging or other diseases, such as frequent leg edema in association with peripheral venous insufficiency in the elderly, might obscure the typical signs observed in younger patients. Age-related crackles in elderly patients with cardiovascular disease might 
be mistakenly diagnosed as a sign of heart failure deterioration, and a danger exists when diuretic therapy is inappropriately instituted. ${ }^{4,14}$ Elderly patients with chronic heart failure and previous decompensation often have persistent pulmonary crackles, even during stable periods, ${ }^{22}$ associated in some cases with the agerelated phenomena, not heart failure status. Whether crackles are related to cardiac dysfunction should be interpreted in light of other clinical tests including, for example, Doppler echocardiography, ${ }^{7,8}$ serum BNP measurement, $17,22,25$ or thoracic CT scans. ${ }^{12,13}$

This study had several limitations. First, more commonly accepted theories ${ }^{26,27}$ for the production of crackles are associated with airway and air-space opening. The pathogenesis of crackles remains unexplained because the CT examination, undertaken in this study to exclude pathologic lung diseases, might not be suitable for exploring the genesis of crackles. Second, our study defined patients with cardiovascular disease as "functionally normal" based on their plasma BNP levels, but this diagnostic test might not be perfect. Because age, sex, and renal function affect serum BNP levels, ${ }^{25,28}$ BNP level cannot be used to strictly define functional cardiac status. In this study, we adopted a cutoff level of BNP at less than $80 \mathrm{pg} / \mathrm{mL}$ for functionally normal hearts because a BNP level of 80 to $100 \mathrm{pg} / \mathrm{mL}$ is widely accepted as a cutoff level for ruling out heart failure status regardless of age..$^{9,10}$ Importantly, logistic regression analysis did not identify BNP levels as the independent predictor of the appearance of crackles in the this study population. Third, left ventricular diastolic function was not examined precisely, and only one index of Doppler-derived E/A ratio ${ }^{6}$ was adopted for identifying diastolic dysfunction in the this study. Thus, some of the participants might belong in the category of stage B cardiovascular status, corresponding to a criterion of diastolic dysfunction with preserved systolic function. ${ }^{6}$ Fourth, this study recruited the study population from highly selected group of patients, which might somewhat limit its generalizability. Finally, despite the importance of crackles for diagnosing acute heart failure decompensation, ${ }^{2,6}$ there is often considerable disagreement among physicians about the actual presence of crackles in individual patients. ${ }^{20}$ In our population $(n=65)$, there was $83 \%$ agreement on interpretation of the presence or absence of crackles.

\section{Implications for Clinicians}

The recognition of age-related crackles is important because such clinically unimportant crackles are so common among elderly patients that, without knowledge of this phenomenon, their existence might interfere with the physician's management of patients with suspected heart failure or presumable pulmonary disease.
To read or post commentaries in response to this article, see it online at http://www.annfammed.org/cgi/content/full/6/3/239.

Key words: Physical examination; auscultation; crackles; respiratory sounds; rales; aging; heart failure

Submitted September 27, 2007; submitted revised December 21, 2007; accepted December 24, 2007.

A version of this study was presented in part at the 73rd annual international scientific assembly of the American College of Chest Physicians, October 20-25, 2007.

\section{References}

1. Kannel WB, Belanger AJ. Epidemiology of heart failure. Am Heart J. 1991;121(3 Pt 1):951-957.

2. Ingram RH Jr, Braunwald E. Pulmonary edema: cardiogenic and

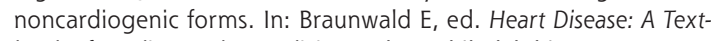
book of Cardiovascular Medicine. Vol. 1. Philadelphia, PA: WB Saunders Co; 1980:571-589.

3. Cabot RC. Physical Diagnosis. 6th ed. New York, NY: William Wood \& Company; 1915:160.

4. Workum P, Holford SK, Delbono EA, Murphy RLH. The prevalence and character of crackles (rales) in young women without significant lung disease. Am Rev Respir Dis. 1982;126(5):921-923.

5. Thacker RE, Kraman SS. The prevalence of ausculatory crackles in subjects without lung disease. Chest. 1982;81(6):672-674.

6. Hunt SA. ACC/AHA 2005 guideline update for the diagnosis and management of chronic heart failure in the adult: a report of the American College of Cardiology/American Heart Association Task Force on Practice Guidelines (W/riting Committee to Update the 2001 Guidelines for the Evaluation and Management of Heart Failure). J Am Coll Cardiol. 2005;46(6):e1-e82.

7. Ellis K, Ziada KM, Vivekananthan D, et al. Transthoracic echocardiographic predictors of left atrial appendage thrombus. Am J Cardiol. 2006;97(3):421-425.

8. Persson $H$, Lonn $E$, Edner $M$, et al. for the Investigators of the CHARM Echocardiographic Substudy-CHARMES. Diastolic dysfunction in heart failure with preserved systolic function: need for objective evidence. J Am Coll Cardiol. 2007;49(6):687-694.

9. Dao Q, Krishnaswamy P, Kazanegra R, et al. Utility of B-type natriuretic peptide in the diagnosis of congestive heart failure in an urgent-care setting. J Am Coll Cardiol. 2001;37(2):379-385.

10. Mueller C, Laule-Kilian K, Frana B, et al. The use of B-type natriuretic peptide in the management of elderly patients with acute dyspnoea. J Intern Med. 2005;258(1):77-85.

11. Lewin S, Goldberg L, Dec W. The spectrum of pulmonary abnormalities on computed chest tomographic imaging in patients with advanced heart failure. Am J Cardiol. 2000;86(1):98-100.

12. American Thoracic Society. Idiopathic pulmonary fibrosis: diagnosis and treatment: international consensus statement. Am J Respir Crit Care Med. 2000;161(2 Pt 1):646-664

13. Zompatori M, Bnà C, Poletti V, et al. Diagnostic imaging of diffuse infiltrative disease of the lung. Respiration. 2004;71(1):4-19.

14. Loudon R, Murphy RLH. Lung sounds. Am Rev Respir Dis. 1984; 130(4):663-673.

15. Fraser RG, Pare JAP. Diagnosis of Diseases of the Chest. 2nd ed. Philadelphia, PA: WB Saunders; 1977:289.

16. Stevenson LW, Perloff JK. The limited reliability of physical signs for estimating hemodynamics in chronic heart failure. JAMA. $1989 ; 261(6): 884-888$. 
17. Kataoka H. Relation of body fluid status to B-type natriuretic peptide levels in chronic heart failure patients during long-term followup. Clin Cardiol. 2006;29(10):457-461.

18. Byrne J, Davie AP, McMurray JJV. Clinical assessment and investigation of patients with suspected heart failure. In: Stewart S, Moser DK, Thompson DR, eds. Caring for the Heart Failure Patient: A Textbook for the Health Care Professional. London: Martin Dunitz; 2004:75-91.

19. Davie AP, Francis CM, Caruana L, Sutherland GR, McMurray JJV. Assessing diagnosis in heart failure: which features are any use? QJM. 1997;90(5):335-339.

20. Gadsbøll N, Høilund-Carlsen PF, Nielsen GG, et al. Symptoms and signs of heart failure in patients with myocardial infarction: reproducibility and relationship to chest X-ray, radionuclide ventriculography and right heart catheterization. Eur Heart J. 1989;10(11):1017-1028.

21. Rohde LE, Beck-da-Silva L, Goldraich L, et al. Reliability and prog nostic value of traditional signs and symptoms in outpatients with congestive heart failure. Can J Cardiol. 2004;20(7):697-702.
22. Kataoka H. Utility of thoracic sonography for follow-up examination of chronic heart failure patients with previous decompensation. Clin Cardiol. 2007;30(7):336-341.

23. Tresch DD. The clinical diagnosis of heart failure in older patients. J Am Geriatr Soc. 1997;45(9):1128-1133.

24. Rich MW. Office management of heart failure in the elderly. Am J Med. 2005;118(4):342-348.

25. Boomsma F, van den Meiracker AH. Plasma A- and B-type natriuretic peptide: physiology, methodology and clinical use. Cardiovasc Res. 2001;51(3):442-449.

26. Forgacs P. Crackles and wheeze. Lancet. 1967;2(7508):203-205.

27. Fredberg JJ, Holford SK. Discrete lung sounds: crackles (rales) as stress-relaxation quadrupoles. J Acoust Soc Am 1983;73(3):1036-1046.

28. Redfield MM, Rodeheffer RJ, Jacobsen SJ, Mahoney DW, Bailey KR, Burnett JC Jr. Plasma brain natriuretic peptide concentration: impact of age and gender. J Am Coll Cardiol. 2002;40(5):976-982. 